\title{
COMPUTATIONALLY EFFICIENT VERSION OF THE DECISION FEEDBACK EQUALIZER
}

\author{
Rajarshi Gupta, Kiran, Edward A Lee \\ University of California, Berkeley \\ $\{$ guptar, mantu, eal\}@eecs.berkeley.edu
}

\begin{abstract}
We propose a computationally efficient version of the Decision Feedback Equalizer (DFE) and compare its performance with the conventional DFE. The proposed equalizer requires fewer taps than the conventional one. This reduces the computational load proportionally and leads to faster adaptation. Identical performance of the two structures in terms of probability of error is also demonstrated using both theoretical and simulation results.
\end{abstract}

\section{INTRODUCTION}

The Decision Feedback Equalizer (DFE) has been used and studied $[1,2,3,4,7,9]$ for so long that it is surprising to find new improvements for it. Nonetheless, we have identified an alternative structure with essentially equivalent performance and between 0 to $50 \%$ reduction in computation as compared to the standard and widely used structure. This is accomplished by using proportionally fewer taps than before to deal with the same channel.

The DFE is commonly split into a forward equalizer and a feedback equalizer. Our analysis builds upon the linear prediction interpretation of the DFE as proposed by Messerschmitt [5]. Computational complexity and speed of adaptation are crucial considerations for a DFE, especially for rapidly varying channels, and the proposed structure is able to achieve improvements in both the aspects.

The paper is outlined as follows. Sections 2 and 3 describe the standard implementation of the DFE and the proposed structure, respectively. In Section 4, we present the simulation model used and the results generated thereof. The advantages and disadvantages of the proposed structure over the currently used one are summarized in Section 5, before we close with a conclusion.

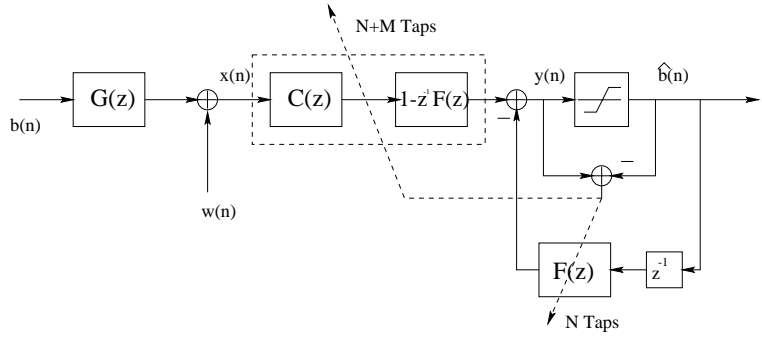

Figure 1: The Decision Feedback equalizer under the current implementation.

\section{STANDARD IMPLEMENTATION}

The channel through which the information is transmitted introduces inter-symbol interference (ISI). In this paper, we consider a discrete-time baseband equivalent of the channel, $G(z)$. In addition to this distortion, the received signal is corrupted by noise, modeled as additive white gaussian noise.

The standard implementation of the DFE is described in $[4,9]$. Fig. 1 shows a block diagram of the DFE, with the separate forward and feedback equalizer paths. Under Messerschmitt's linear prediction interpretation, the received signal $x(n)$ is passed through the zero forcing equalizer $C(z)$ and the prediction error filter $\left(1-z^{-1} F(z)\right)$. The combination of the two forms the forward equalizer which removes the precursor ISI, without noise enhancement. The other filter in the DFE is the feedback equalizer, $F(z)$, which removes the postcursor ISI. As observed in [5], F(z) can be derived from the linear predictor for the colored noise at the output of $C(z)$. The zero forcing equalizer exists for a wide variety of channels, as demonstrated in [4]. For a time varying channel, the tap values of both the forward and feedback equalizers are made adaptive, using the decision-directed technique.

If the channel equalization requires $M$ taps and the feedback path has $N$ taps, then the feedforward path will have $N+M$ taps, making the total number taps 


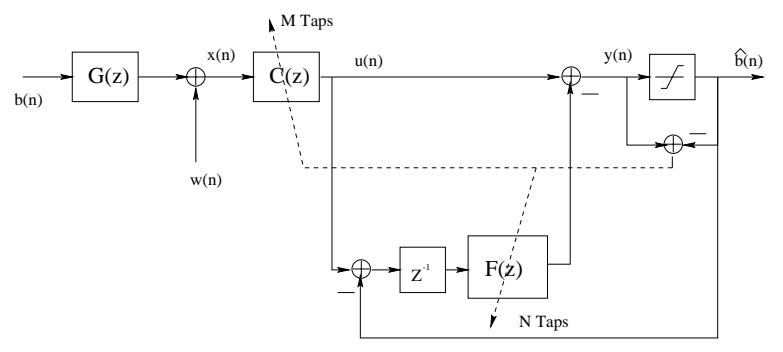

Figure 2: The proposed equalizer.

required $2 N+M$. The choice of $M$ (the number of taps for the zero forcing equalizer) is governed by the channel characteristics while $N$ (the number of taps in the linear predictor) depends on the rate of decay of the zero forcing equalizer's impulse response. For example, if the channel is modeled as a $k$-pole filter, then $M=k+1 ; N$ is ideally infinite, but is truncated to a reasonable length depending on the pole locations, for practical implementation.

\section{PROPOSED STRUCTURE}

An alternative structure for the DFE is presented in Fig. 2.

The received signal $x(n)$ is first fed through the zero forcing equalizer $C(z)$. This filter removes the ISI completely, so the output $u(n)$ is the transmitted signal corrupted by colored noise. Since we are using a simple threshold for the decision device, best performance is achieved when the background noise is white. So the next step is to whiten the noise, without affecting the signal. Assuming the tap values are well adapted to the channel (having used a training sequence during initialization), the estimate $\hat{b}_{n}$ is correct with high probability. Th approximation of the colored noise given by $u(n)-\hat{b}(n)$ is fed into the linear predictor $F(z)$ to remove the correlation in the colored noise. The output $y(n)$ is then the desired signal corrupted by white noise, and is thresholded to yield $\hat{b}_{n}$. Thus we have a channel equalizer $C(z)$ and a predictor $F(z)$ to achieve the effect of the DFE described in Section 2. The system is made adaptive using the standard decision-directed method and its performance estimated in terms of the probability of error in the quantized signal.

The channel equalizer needs $M$ taps while the predictor needs $N$ taps, making a total of $N+M$ taps for equalization. The choice of $N$ and $M$ are governed by the same considerations as outlined in Section 2. This provides us with a reduction of $N$ taps over the conventional DFE implementation. The fractional gain in the number of taps is thus $\frac{N+M}{2 N+M}$. In the worst case, for $N \ll M$, there is little reduction in number of taps.

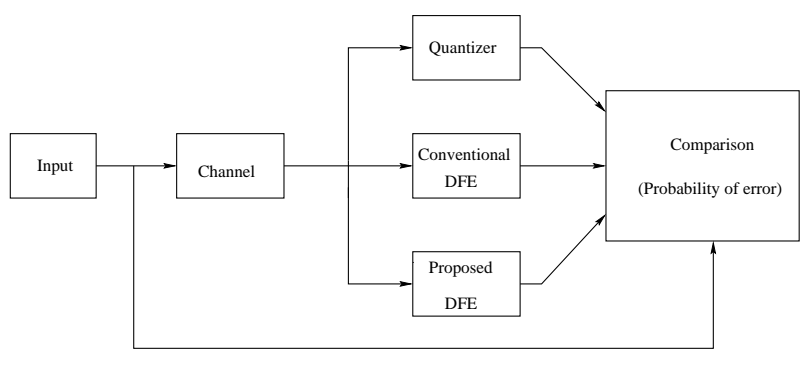

Figure 3: The simulation model.

However, when $N \gg M$, we have as much as $50 \%$ reduction in the number of taps. For a typical situation when $N \simeq M$ the improvement achieved is $33 \%$.

We consider the equivalence of the two zero-forcing structures:

$Y_{D F E}(z)=X(z) C(z)\left[1-z^{-1} F(z)\right]+z^{-1} F(z) \hat{b}_{D F E}(z)$

$Y_{N E W}(z)=X(z) C(z)-z^{-1} F(z)\left[X(z) C(z)-\hat{b}_{N E W}(z)\right]$

If the probability of error is sufficiently low, then we have $\hat{b}_{D F E}(n)=\hat{b}_{N E W}(n)=\hat{b}(n)$ and therefore $Y_{D F E}(z)=Y_{N E W}(z)$. Furthermore, in a noiseless situation, when the received signal is distorted by the dispersive channel alone, we have $\hat{b}(n)=b(n)$, showing that both the structures are zero-forcing and achieve the same performance.

\section{SIMULATION}

To verify the theoretical results, we set up a simulation model for the system, using the Ptolemy simulation environment [http://ptolemy.eecs.berkeley.edu].

In the simulation model illustrated in Fig. 3, we model the input bit stream to be binary antipodal. It passes through a channel which is modeled as an allpole filter, corrupted by additive white gaussian noise. The outputs from the conventional DFE and the proposed DFE are then compared with respect to their rates of convergence and probabilities of error.

The signal to noise ratio (SNR) for the 2-pole channel is varied over the range 1 to $15 \mathrm{~dB}$ and the probability of error is computed for each of the structures. The results plotted in Fig. 4 show that the DFE and the newly proposed algorithm perform almost identically, when there are enough taps to match the chosen AR model for the channel. The plot also includes the unequalized case, which justifies the use of the equalizer.

The theoretical limit for the probability of error in the case of binary antipodal signaling over an additive white gaussian noise channel is given by $P_{e}=Q\left(\sqrt{\frac{\gamma b}{2}}\right)$, 


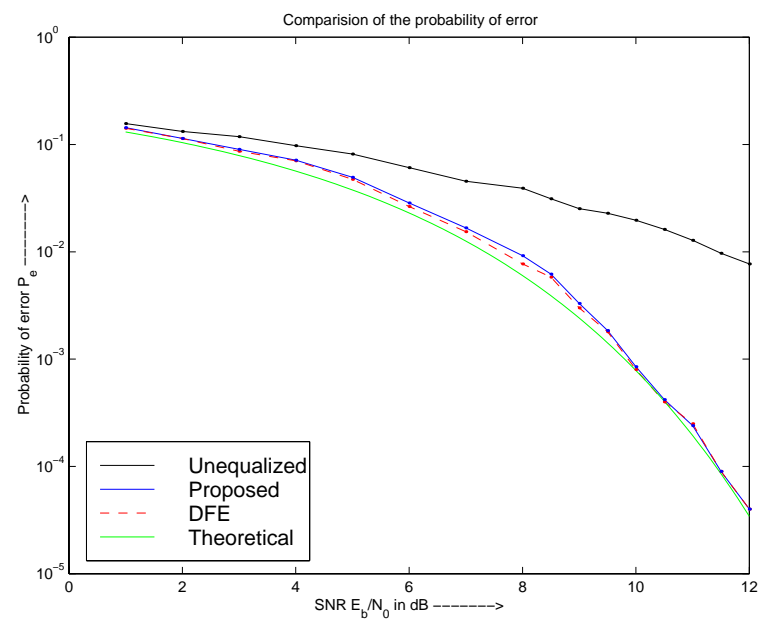

Figure 4: A Comparison of the probability of error.

where $\gamma_{b}$ is the SNR per bit [9]. As seen from the plot, the performance of both the DFE and the proposed structure are very close to the theoretical limit, leaving little scope for improvement in this simple channel model.

In addition to the above simulations, the following cases of channels were also considered,

- Minimum-phase FIR model: The channel is modeled to be an 'All Zero' filter, with all zeros inside the unit circle. Here the channel is invertible and the LMS filters are ideally infinite impulse response filters. But in practice, a large enough number of taps (depending on the location of the zeroes of the channel) is sufficient to achieve performance that is close to the theoretical limit. In the simulations, 12 taps used for a channel model having a 3 tap response was seen to have a performance very close to the theoretical limit.

- Non-Minimum phase FIR model : The channel is modeled to be an 'All Zero' filter, with not all zeros inside the unit circle. The channel is definitely not invertible but its equalizer exists due to the existence of the zero forcing equalizer [4]. The simulations were performed with a channel filter having one zero outside the unit circle and the other inside. It was seen that after an initial transient period, the probability of error could be brought arbitrarily close to the theoretical limit by increasing the number of taps in the LMS filter. In the example chosen, the LMS filters with 12 taps showed results comparable to the ones stated above.

- ARMA model : The channel is a 'Pole-Zero' IIR filter. The impulse response of the channel is chosen to be of the form $r^{n} \cos \left(\omega_{0} n\right) u(n)$ with $\omega_{0}=\frac{\pi}{4}$ and $r=\frac{1}{\sqrt{2}}$. This gives a filter with 2 poles and a zero. As expected, both the equalizers perform identically and close to the theoretical limit.

\section{ADVANTAGES AND DISADVANTAGES}

The proposed equalizer has been shown to perform identically to the DFE in Section 4 in terms of probability of error. The new structure is however preferable due to a number of advantages explained below.

- The proposed implementation requires only $N+$ $M$ taps compared to the $2 N+M$ taps in the conventional DFE (Sections 2 and 3). A saving of $N$ taps per sample is a significant reduction in complexity.

- The update stepsize $\beta$ of an adaptive filter is constrained by $0<\beta<\frac{1}{K \sigma^{2}}$ where $K$ is the number of taps and $\sigma^{2}$ is the variance of the input random process [3]. The stepsize governs the rate of convergence, with a larger stepsize resulting in a faster rate of convergence. In both the structures, $F(z)$ has the same number of taps and hence its convergence is identical in both. But the forward equalizer in the DFE has $N+M$ taps implying $0<\beta_{D F E}<\frac{1}{(N+M) \sigma^{2}}$ while the channel inversion in the proposed structure has $N$ taps and thus $0<\beta_{N E W}<\frac{1}{N \sigma^{2}}$. This means that we can choose a larger $\beta$ for the proposed structure, ensuring faster convergence rates and better stability. The proposed structure thus has better adaptation to time-varying channels.

- For a large class of channels, the channel equalizer has an impulse response that looks like $C(z)=$ $C_{1}(z)+z^{-L} C_{2}(z)$. Such situations are common in a closed room kind of environment and in echo paths and the difference in the number of tap updates becomes even more apparent here.

Suppose $C_{1}(z)$ requires $M_{1}$ taps and $C_{2}(z)$ requires $M_{2}$ taps, and $L \gg M_{1}, M_{2}$. In the proposed structure, we would have a parallel implementation of two filters (Fig. 5), which means that the implementation would take $M_{1}+M_{2}$ taps.

In the conventional DFE, we have the channel equalizer $C(z)$ convolved by another filter. Convolution being a spreading operation in time domain, we can expect to require more tap updates. In particular, for the system in Fig. 6, we 


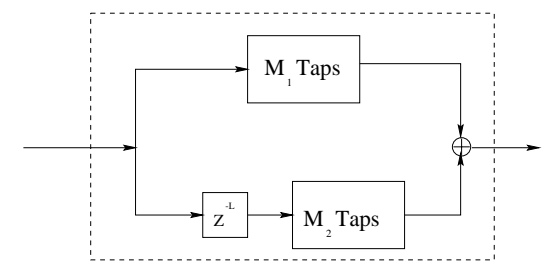

Figure 5: Channel equalizer in the proposed structure.

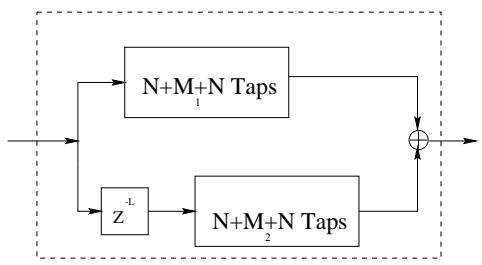

Figure 6: Channel equalizer in conventional DFE.

would require $\left(N+M_{1}+N\right)+\left(N+M_{2}+N\right)=$ $4 N+M_{1}+M_{2}$ updates. Thus in this practical situation, we save up to $4 N$ taps, instead of the previously achieved gain of $N$ taps.

- The tap values in the proposed equalizer are spread over a much narrower range than in the conventional DFE. In the feed-forward part of the conventional equalizer, the zero forcing equalizer $C(z)$ is convolved with $1-z^{-1} F(z)$. The convolution increases the spread in the range of tap values. This poses a problem with quantization of tap values [8], which is reduced in the proposed structure.

However, the proposed structure for the DFE fails to perform at par for certain other situations.

- The input to the linear predictor $F(z)$ in the conventional structure is a symbol sequence (Fig. 1). Hence, in VLSI implementations of the DFE, asymmetric multipliers can be used to achieve significant hardware savings. In the proposed structure (Fig. 2), the input to $F(z)$ is spread over the entire continuum due to the presence of noise (rather than being confined to the symbol set). So, the asymmetric multipliers cannot be used and these savings are lost.

However, in the case of programmable processor implementations, where special hardware is not used, the above problem does not arise.

\section{CONCLUSION AND FUTURE WORK}

We have outlined our efforts in proposing a computationally efficient version of the Decision Feedback Equalizer. In this paper, we presented the proposed DFE and compared it with the conventional DFE. The proposed equalizer requires fewer taps and hence has fewer computations (for filtering). Moreover, it also has a faster adaptability owing to its higher convergence rate. The two structures perform identically in terms of probability of error, as shown using both theoretical and simulations results. Finally, we outlined the advantages and disadvantages of using the proposed DFE over the conventional one.

The issue of the rate of convergence requires a much more detailed analysis than accorded in this paper. The applications of the proposed structure in special situations like the fractionally spaced DFE also need to be analyzed.

\section{ACKNOWLEDGMENTS}

We would like to express our thanks to David Messerschmitt for his guidance and invaluable advice.

\section{REFERENCES}

[1] Belfore, C.A. and Park, J.H. "Decision Feedback Equalization",Proceedings of the IEEE, Vol. 67, No.8, p.1143-1156, Aug. 1979.

[2] George, D.A., Baowen, R.R., and Storey, J.R. "An adaptive decision feedback equalizer", IEEE Trans. Commun. Tech., Vol. COM-19, June 1971, p.281-293.

[3] Haykin, S., "Adaptive filter theory", Prentice Hall, Third edition, 1996.

[4] Lee, E.A. and Messerschmitt, D.G., "Digital Communication", Kluwer Academic Publishers, Second Edition, 1994.

[5] Messerschmitt, D.G., "A geometric theory of intersymbol interference, Part I", Bell System Tech. J., Vol. 52, no. 9, Nov. 1973, p.1483-519.

[6] Messerschmitt, D.G., "A geometric theory of intersymbol interference, Part II", Bell System Tech. J., Vol. 52, no. 9, Nov. 1973, p.1521-39.

[7] Messerschmitt, D.G. "Comments on 'Bounds on probability of error in decision feedback equalizer' ". IEEE Trans. on Aerospace and Electronic Systems, Vol. AES-14, no. 2, March 1978, p.373-5.

[8] Oppenheim, A.V. and Schafer, R.W., "Discretetime signal processing", Prentice Hall, 1989.

[9] Proakis, J.G., "Digital Communications", McGraw-Hill,Inc, Third edition, 1995.

[10] Salz, J., "Optimum mean-square decision feedback equalization", Bell System Tech. J., Oct 1973, p.1341-74. 